\title{
The Efficacy of Laparoscopic Ovarian Electrocautery on Infertile Patients with Polycystic Ovary Syndrome (PCOS) and Resistance to Ovulation Induction Treatment
}

\section{ART ICLE INF O}

\section{Article Type}

Original research

\section{Authors}

Saremi A.T.* $M D$

Tarazi B. ${ }^{1} M D$,

Ghanbari F. ${ }^{2} M S c$

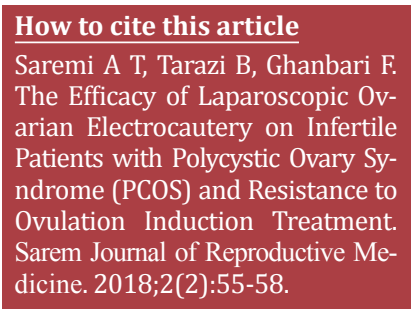

*"Sarem Fertility \& Infertility Research Center (SAFIR)" and "Sarem Cell Research Center (SCRC)", Sarem Women's Hospital, Tehran, Iran ${ }^{1}$ Aban Hospital, Tehran, Iran

${ }^{2}$ Sarem Women's Hospital, Tehran, Iran

\section{Correspondence}

Address: Sarem Women's Hospital, Basij Square, Phase 3, Ekbatan Town, Tehran, Iran. Postal Code: 1396956111

Phone: +98 (21) 44670888

Fax: +98 (21) 44670432

saremiat@yahoo.com

\section{Article History}

Received: February 4, 2017

Accepted: May 14, 2017

ePublished: June 15, 2018

\section{A B S T RA C T}

Aims Polycystic ovary syndrome (PCOS) is one of the clinical conditions associated with chronic anovulation and infertility. Non-surgical procedures, including ovulation induction drugs or surgical procedures, are used to treat it. The objective of this study was to investigate the efficacy of laparoscopic ovarian electrocautery on infertile patients with PCOS and resistance to ovulation induction treatment.

Materials \& Methods This experimental study was conducted on 183 infertile female patients with PCOS and resistance to drug treatment that referred to Sarem women's hospital (Tehran, Iran) during a five-year period. Sampling was done by sequential census. For all patients, ovarian laparoscopy and electrocautery were carried out and all of them were subjected to ovulation induction protocols. Ovarian response was evaluated along with variables such as age, duration of infertility, menstrual status, the levels of LH and FSH, hirsutism, and time interval between laparoscopy and ovulation. Data were analyzed by Chi-square and independent $\mathrm{t}$ tests using SPSS software.

Findings The ovulation rate was found as $81.4 \%$ among the patients, but only in $26 \%$ of those patients pregnancy completed successfully. There was no significant relationships between ovulation with the levels of LH, FSH and LH/FSH ratio ( $p>0.05)$. The relationship between ovarian response and postoperative treatment protocol was significant $(\mathrm{p}=0.0001)$, which indicated a substantial treatment response for any ovulation induction with clomid.

Conclusion Laparoscopic ovarian electrocautery is an appropriate treatment for infertile patients with PCOS and resistance to ovulation induction treatment.

Keywords Ovarian Electrocautery; Polycystic Ovarian Syndrome; Infertility

\section{I T A T I O N L I N KS}

[1] Epidemiology of infertility and polycystic ovarian disease: Endocrinological and demographic studies [2] Polycystic ovary syndrome: What is the role of obesity? [3] Amenorrhea associated with bilateral polycystic ovaries [4] Laparoscopic treatment of polycystic ovaries with unipolar forceps cautery [5] Effect of laparoscopic electrocautery of ovaries on ovarian response and fertility in poor response PCOS patients [6] Laparoscopic procedures for treatment of infertility related to polycystic ovarian syndrome [7] The effectiv eness of laparoscopic electrocautery in clomip hene citrate resistant patients with p olycystic ovary syndrome in relation to ovarian size [8] Polycystic Ovarian syndrome: Diagnosis and management [9] Malignancy associated changes in Cervical Smears: Systematic changes in cytometric features with the grade of dysplasia [10] Insulin stimulates androgen accumulation in incubations of ovarian stroma obtained from women with hyperandrogenism [11] Insulin administration alters gonadal steroid metabolism independent of changes in gonadotropin secretion in insulin-resistant women with the polycystic ovary syndrome [12] Insulin resistance and the polycystic ovary syndrome: Mechanism and implications for pathogenesis [13] Polycystic ovaries treated by laparoscopic laser vaporization [14] A double blind controlled study of the hormonal and clinical effects of bromocriptine in the polycystic ovary syndrome [15] Hormonal response to electrocautery of the ovary in patients with polycystic ovarian disease [16] Effect of laparoscopic ovarian electrocautery on ovarian response and outcome of treatment with gonadotropins in clomiphene citrate-resistant patients with polycystic ovary syndrome [17] A comparison between ultrasound and gynecologic examination for detection of enlarged ovaries in a group of women at risk for ovarian carcinoma [18] Ovulatory cycles, pregnancy outcome and complications after surgical treatment of polycystic ovary syndrome [19] 9 Surgical options in polycystic ovary syndrome patients who do not respond to medical ovulation induction [20] Laparoscopic treatment of polycystic ovaries with insulated needle cautery: A reappraisal [21] Transvaginal ovarian drilling: A new surgical treatment for improving the clinical outcome of assisted reproductive technologies in patients with polycystic ovary syndrome 
سيترات است[5]. ممكن است تعدادى از اين بيماران به كلوميفن

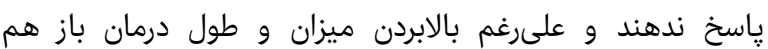

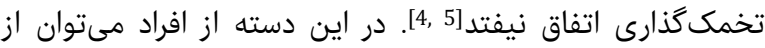

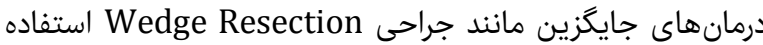

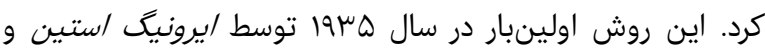

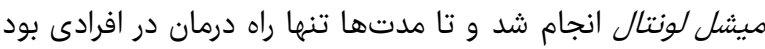

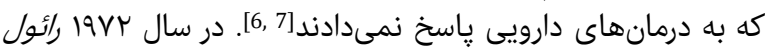

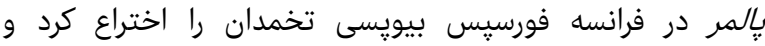
كوتركردن تخمدانها يكى از روشهاى جرانى تخراحى در جهان معرفى اختراع كرد

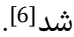

هدف اين مطالعه بررسى اثربخشى الكتروكوترى لإياراسكوييك

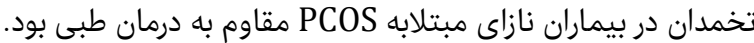

مواد و روشها

در اين مطالعه تجربى

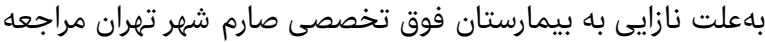

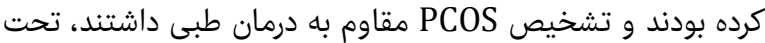

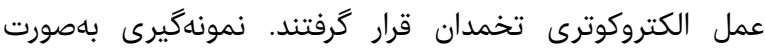

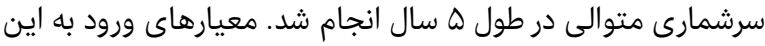

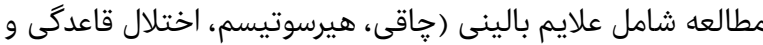

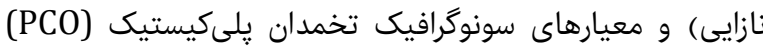

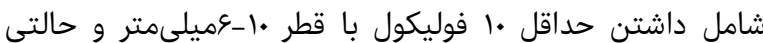

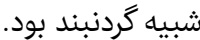

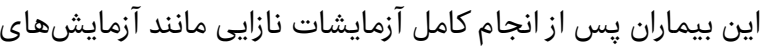

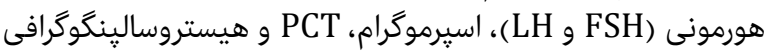

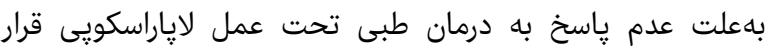
كرفتند. الكتروكوترى از طريق

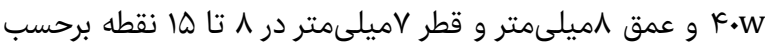

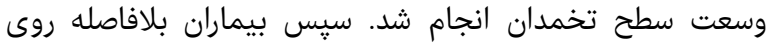

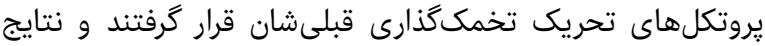

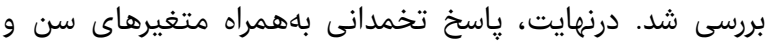

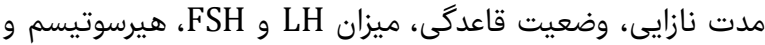

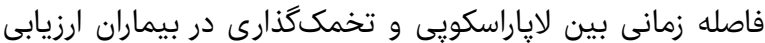

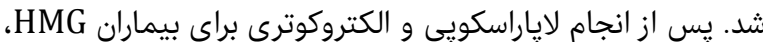

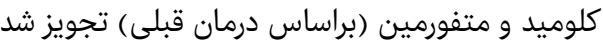

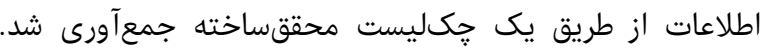

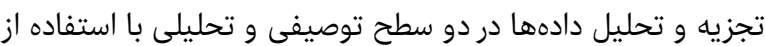

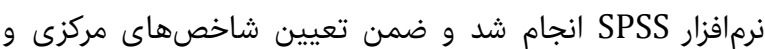

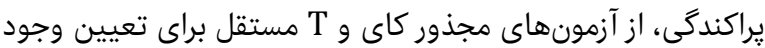
يا عدم وجود ارتباط بين متغيرها و شداى شدآ آن استفاده شد.

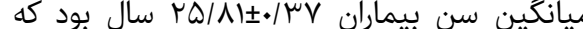

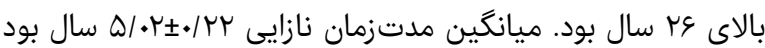

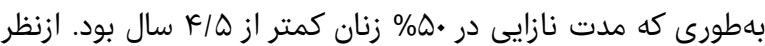

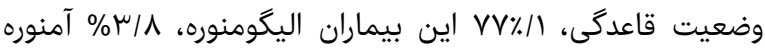

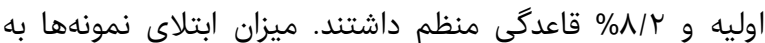

هيرسوتيسم

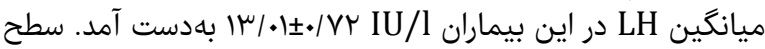

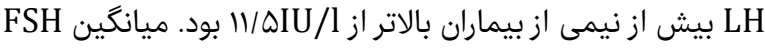

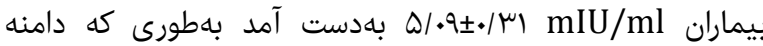

\section{اثربخشى الكتروكوترى لاياراسكوييك تخدمان

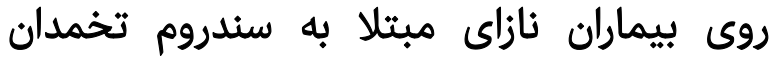 يلىكيستيك (PCOS) مقاوم به درمان}

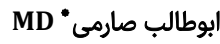

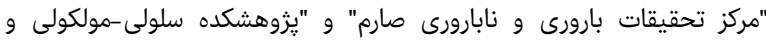

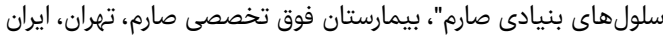 \\ بيتك طرازى بئي \\ بيمارستان آبان، تهران، ايران \\ MSc فريبا قنبرى \\ بيمارستان فوق تخصصى صارم، تهران، ايران
}

جكيده

اهداف: سندروم تخمدان يلىكيستيك (PCOS) يكى از حالتهاى بإى بالينى همراه

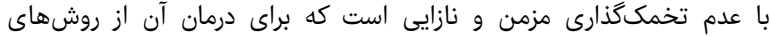

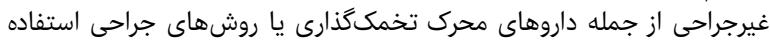

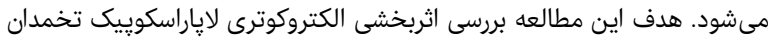

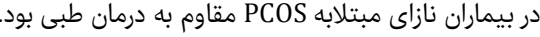

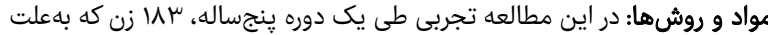

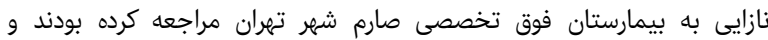

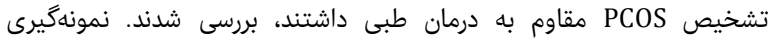

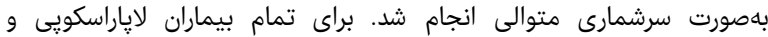

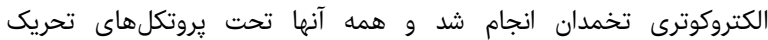

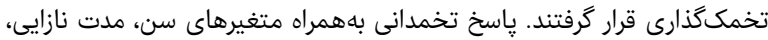

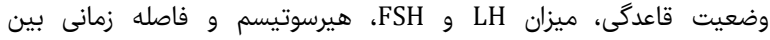

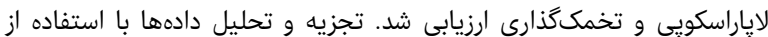

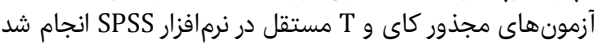

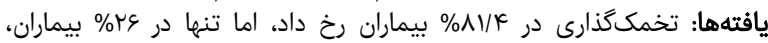

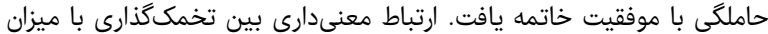

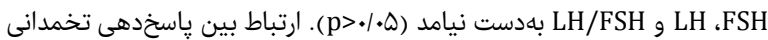

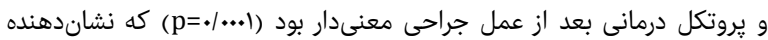

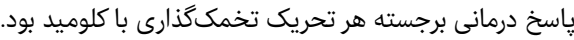

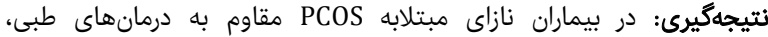

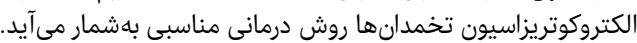

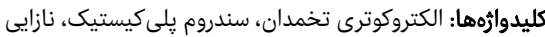

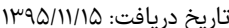

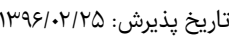

'نويسنده مسئول: saremiat@yahoo.com

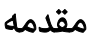

در سال هس19 ميلادى، دو دانشمند آلمانى با نامهاى /يرونيكً

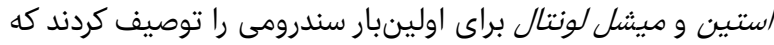

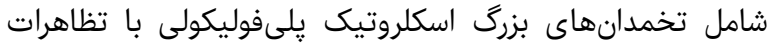

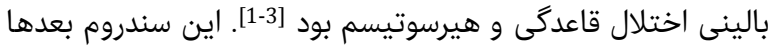

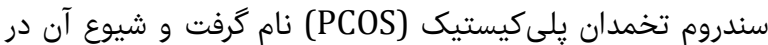

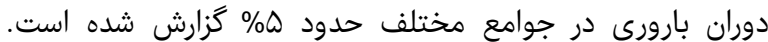

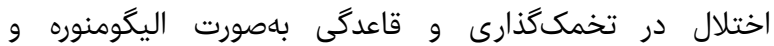

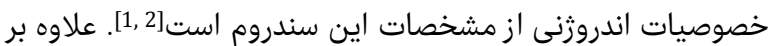

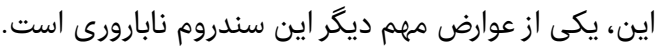

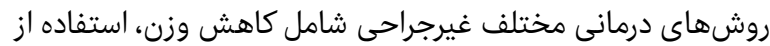

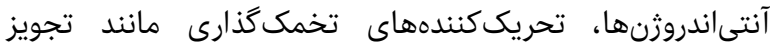

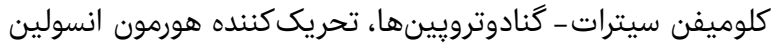

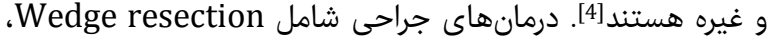

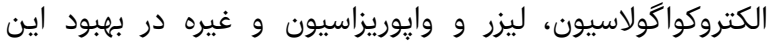
بيماران توصيه شده است كه اولين قدم درمان تجويز 


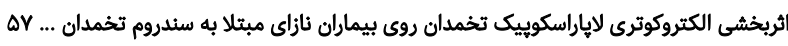

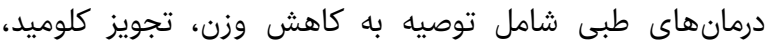

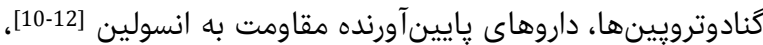

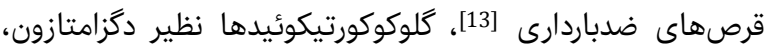

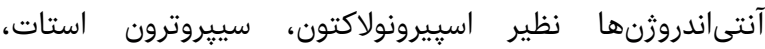

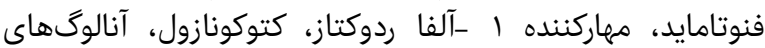

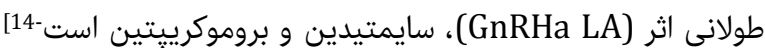

در سال 19 19 در يزوهشى كه توسط استين انجام گرفت، از

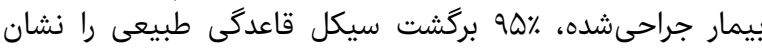

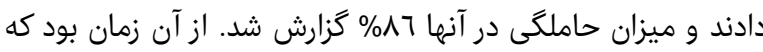

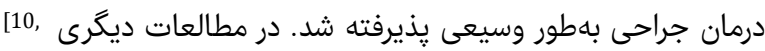

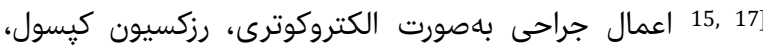

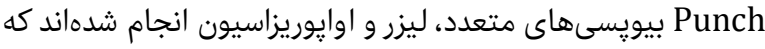

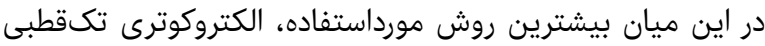

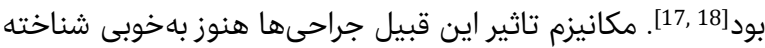

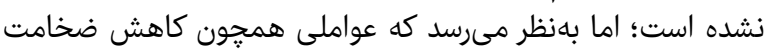

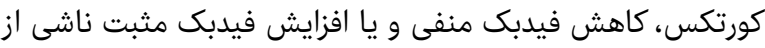

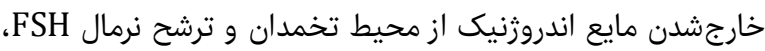

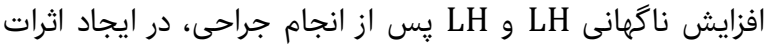

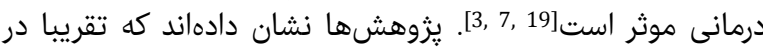

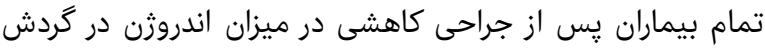

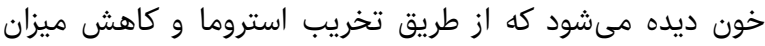

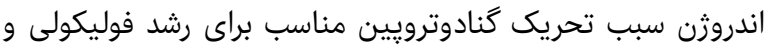

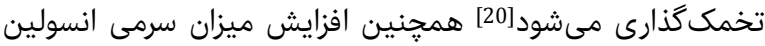

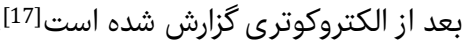

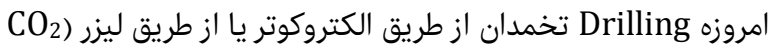

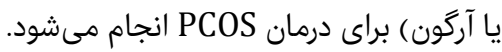

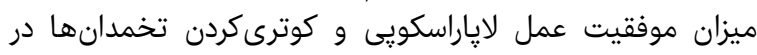

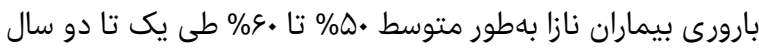

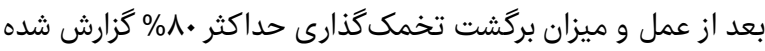

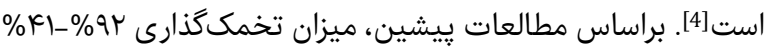

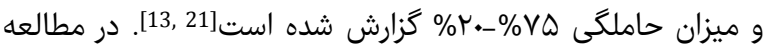

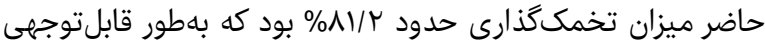

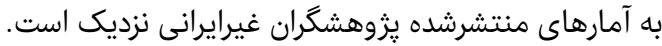

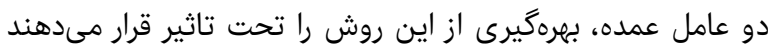

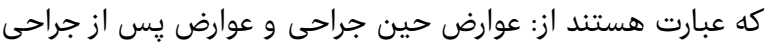

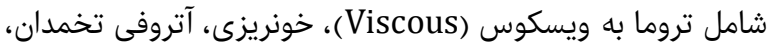

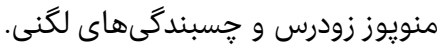

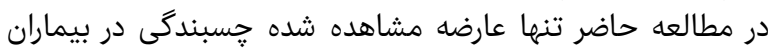

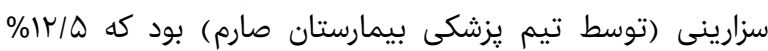

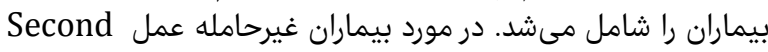
look

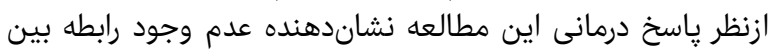

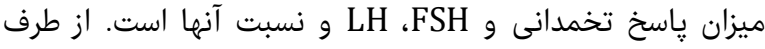

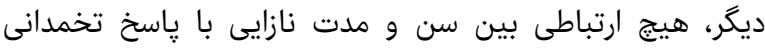

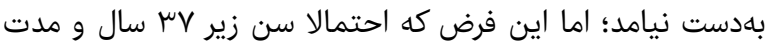

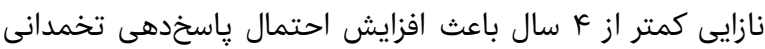

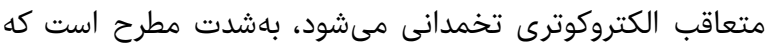

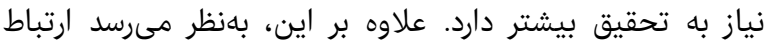

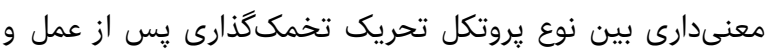

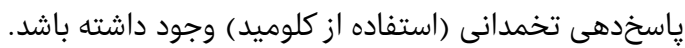

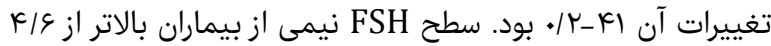

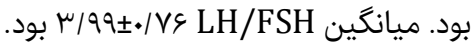

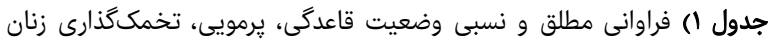

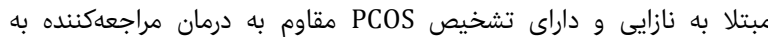

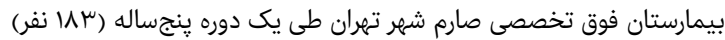

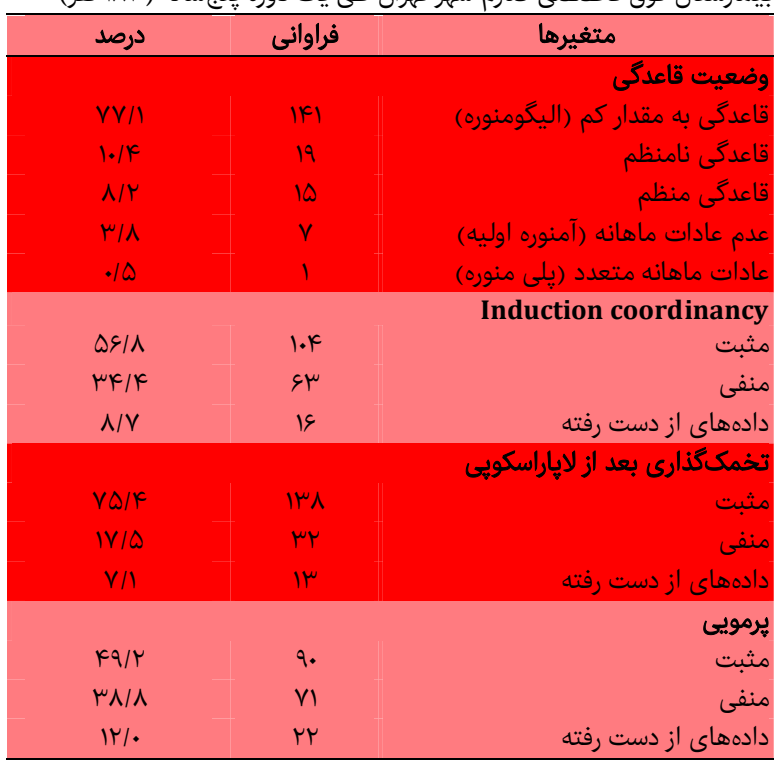

با تجويز HMG، كلوميد و متفورمين به بيماران (براساس درمان

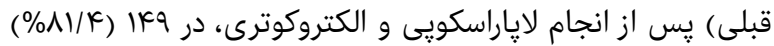

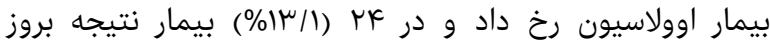

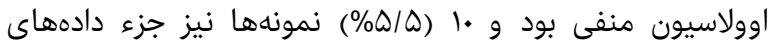

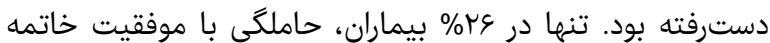

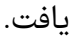

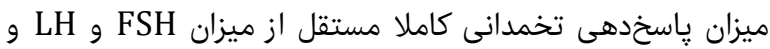

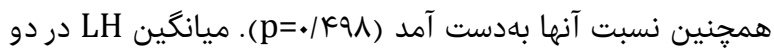

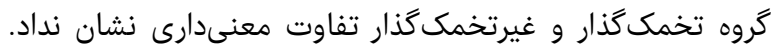

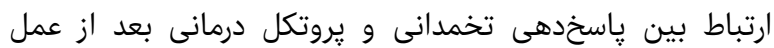

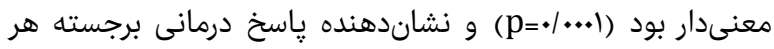

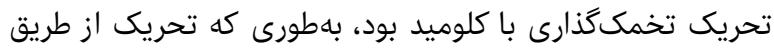
متفورمين، تركيب كلوميد و

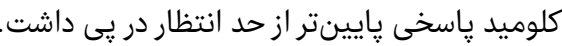

بحث

در اين مطالعه اثربخشى الكتروكوترى لاياراسكوِيكى تخمدان در

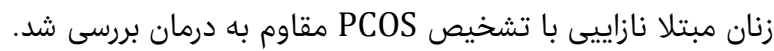

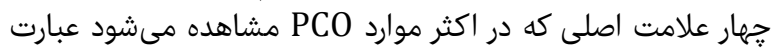

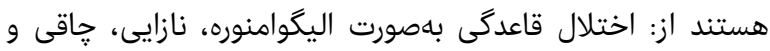

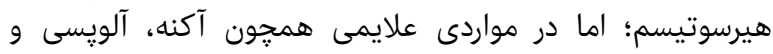

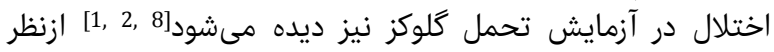

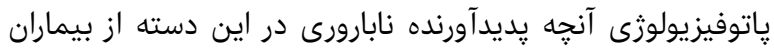

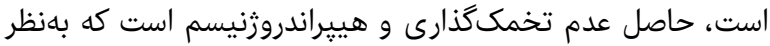

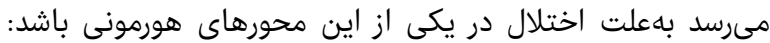

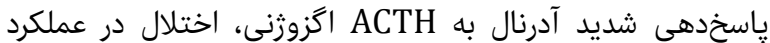

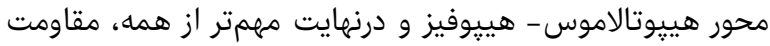

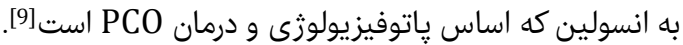

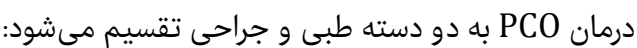


and management. Clin Med Res. 2004;2(1):13-27.

9- Carmina E, Koyama T, Chang L, Stanczyk FZ, Lobo RA. Does ethnicity influence the prevalence of adrenal hyperandrogenism and insulin resistance in polycystic ovary syndrome? Am J Obstet Gynecol. 1992;167(6):1807-12.

10- Barbieri Rl, Makris A, Randall Rw, Daniels G, Kistner $\mathrm{Rw}$, Ryan KJ. Insulin stimulates androgen accumulation in incubations of ovarian stroma obtained from women with hyperandrogenism. J Clin Endocrinol Metab. 1986;62(5):904-10.

11- Dunaif A, Graf M. Insulin administration alters gonadal steroid metabolism independent of changes in gonadotropin secretion in insulin-resistant women with the polycystic ovary syndrome. J Clin Invest. 1989;83(1):23-9.

12- Dunaif A. Insulin resistance and the polycystic ovary syndrome: Mechanism and implications for pathogenesis. Endocr Rev. 1997;18(6):774-800.

13- Daniell JF, Miller W. Polycystic ovaries treated by laparoscopic laser vaporization. Fertil Steril. 1989;51(2):232-6.

14- Buvat J, Buvat-herbaut M, Marcolin G, Racadot A, Fourlinnie JC, Beuscart R, et al. A double blind controlled study of the hormonal and clinical effects of bromocriptine in the polycystic ovary syndrome. J Clin Endocrinol Metab. 1986;63(1):119-24.

15- Aakvaag A, GjøNnæss H. Hormonal response to electrocautery of the ovary in patients with polycystic ovarian disease. $\mathrm{Br} \mathrm{J}$ Obstet Gynaecol. 1985;92(12):1258-64.

16- Farhi J, Soule S, Jacobs HS. Effect of laparoscopic ovarian electrocautery on ovarian response and outcome of treatment with gonadotropins in clomiphene citrateresistant patients with polycystic ovary syndrome. Fertil Steril. 1995;64(5):930-5.

17- Granberg S, Wikland M. A comparison between ultrasound and gynecologic examination for detection of enlarged ovaries in a group of women at risk for ovarian carcinoma. J Ultrasound Med. 1988;7(2):59-64.

18- Campo S. Ovulatory cycles, pregnancy outcome and complications after surgical treatment of polycystic ovary syndrome. Obstet Gynecol Surv. 1998;53(5):297308.

19- Greenblatt E. 9 Surgical options in polycystic ovary syndrome patients who do not respond to medical ovulation induction. Baillière Clin Obstet Gynaecol. 1993;7(2):421-33.

20- Felemban A, Tan SL, Tulandi T. Laparoscopic treatment of polycystic ovaries with insulated needle cautery: A reappraisal. Fertil Steril. 2000;73(2):266-9.

21- Ferraretti AP, Gianaroli L, Magli MC, Iammarrone E, Feliciani E, Fortini D. Transvaginal ovarian drilling: A new surgical treatment for improving the clinical outcome of assisted reproductive technologies in patients with polycystic ovary syndrome. Fertil Steril. 2001;76(4):812-6.
زمانى كه هورمونترايى با شكست موارئ مواجه شود يا در مواردى كه خطر

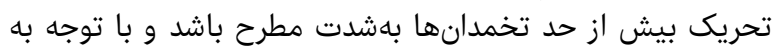

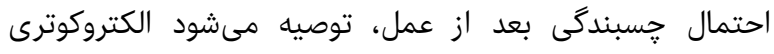
تخمدان از طريق لاياراسكويى انجام شود.

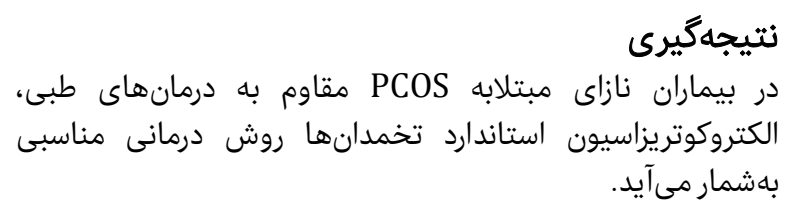

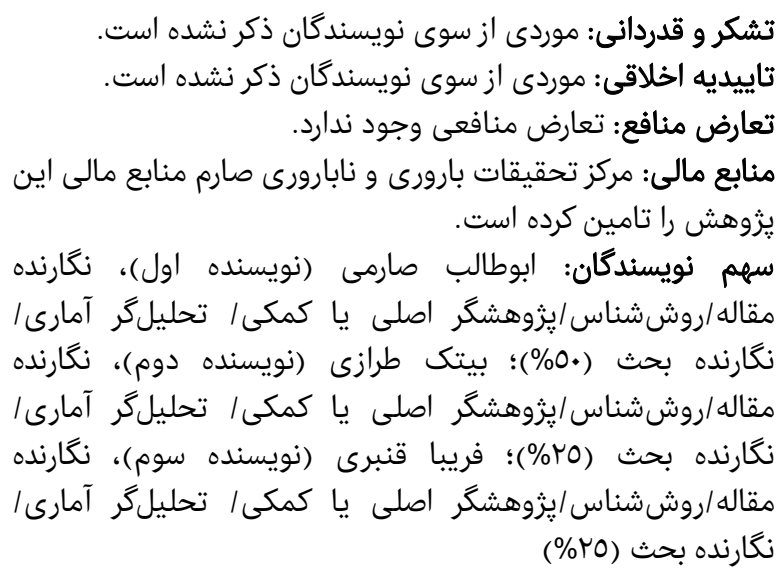

منابع ovarian disease: Endocrinological and demographic studies. Gynecol Endocrinol. 1987;1(3):235-45.

2- Isikoglu $M$, Berkkanoglu $M$, Cemal $H$, Ozgur K. Polycystic ovary syndrome: What is the role of obesity?. In: Allahabadia GN, Agrawal R, editors. Polycystic Ovary Syndrome. United Kingdom: Anshan; 2007. p.157-63.

3- Stein IF, Leventhal ML. Amenorrhea associated with bilateral polycystic ovaries. Am J Obstet Gynecol. 1935;29(2):181-91.

4- Zareian Z, Zareian P. Laparoscopic treatment of polycystic ovaries with unipolar forceps cautery. Razi J Med Sci. 2003;10(34):223-8.

5- Esmailzadeh S, Nazari T. Effect of laparoscopic electrocautery of ovaries on ovarian response and fertility in poor response PCOS patients. Sci J Kurd Univ Med Sci. 2007;12(1):60-5.

6- Cohen J. Laparoscopic procedures for treatment of infertility related to polycystic ovarian syndrome. Hum Reprod Update. 1996;2(4):337-44.

7- Alborzi S, Robati M, Parsanejad M. The effectiv eness of laparoscopic electrocautery in clomip hene citrate resistant patients with $\mathrm{p}$ olycystic ovary syndrome in relation to ovarian size. Med J Islam Repub Iran. 2001;15(3):143-7.

8- Sheehan MT. Polycystic Ovarian syndrome: Diagnosis 